\title{
Tendencias globales en la educación universitaria: Una mirada desde el liderazgo transformacional
}

\section{Global trends in university education: a look from transformational leadership}

Summar Alfredo Gómez Barrios ${ }^{1}$

INFORMACIÓN DEL

ARTÍCULO

Fecha de recepción: 5 de Febrero de 2018.

Fecha de aceptación: 15 de Mayo de 2018.

1 Doctor en Gerencia y Magíster en Gerencia Empresarial, Universidad Central de Venezuela. Docente y Sub director del Instituto de Investigaciones Científicas y Tecnológicas de la Universidad Iberoamericana del Ecuador. E-mail: summar.gomez@gmail.com
CITACIÓN: Gómez Barrios, S. A. (2018). Tendencias globales en la educación universitaria: Una mirada desde el liderazgo transformacional. Podium, 33, 69-78.

doi:10.31095/podium.2018.33.7

ENLACE DOI:

http://dx.doi.org/10.31095/podium.201 8.33 .7

\section{Resumen}

Este artículo tiene por objeto dar a conocer el estado del arte de las tendencias globales en la educación universitaria con una mirada desde el liderazgo transformacional. Este ensayo de carácter argumentativo expone los grandes problemas que impactan a la humanidad, los cuales requieren ser abordados -además del Estado y las empresas-, por las universidades, dado que han tenido un rol importante en su solución, haciendo cambios profundos para responder a las demandas de la sociedad. Se concluye que para garantizar los cambios y alinearse con las tendencias globales en educación universitaria, es necesario que estas instituciones modifiquen la manera de conducirse, a través de un liderazgo que permita transformar la concepción que se tiene acerca de la realidad, a los fines de poder alinearse con las tendencias globales en este importante sector.

Palabras Clave:

Tendencias globales, educación universitaria, liderazgo transformacional, Estado, empresas.

Clasificación JEL: I21, I23, I28, O5.

\section{Abstract}

This article aims to publicize the state-of-the-art in global trends in university education looked at from the viewpoint of transformational leadership. This essay is of an argumentative character; it presents the great problems that impact humanity, which need to be addressed, not only by the state and by companies, but also by universities, given that they have played an important role in their solution; they have made profound changes to respond to the demands of society. It is concluded that, to guarantee changes and align with global trends in university education, these institutions need to modify their leadership and the way in which they conduct themselves in this important sector.

Keywords:

Global trends, university education, transformational leadership, State, companies.

JEL Classification: I21, I23, I28, O5.

\section{9}

PODIUM No. 33, Junio 2018, pp. 69-78

Universidad Espíritu Santo - UEES

ISSN: 1390-5473 e-ISSN: 2588-0969 


\section{Introducción}

Las tendencias en la educación universitaria en el orden social, económico, político, científico $\mathrm{y}$ tecnológico a escala planetaria han generado una gran incertidumbre $y$ revisión del modo de concebir la realidad académica e investigativa. Las exigencias hablan de establecer un nuevo modelo de gestión universitaria que conserve suficiente flexibilidad y que al mismo tiempo busque romper las líneas ya establecidas sobre bases tradicionales entre el Estado, las universidades, el sector productivo y la sociedad. En este sentido, la globalización ha sido una extraordinaria tribuna para las universidades, a los fines de "enriquecer sus patrimonios de saberes, para fecundar con la transferencia de las mejores prácticas la multiplicidad de experiencias que están portadas en la inmensa variedad de universidades en el mundo" (Lanz y Ferguson, 2011, p. 181).

Es evidente entonces la necesidad de concebir una educación universitaria encaminada a facilitar un aprendizaje que incentive las capacidades interpretativas del estudiante, que se vincule con el mundo de una manera diferente, y que se involucre directamente en la búsqueda de información y conocimientos en ambientes globalizados. En sí, una educación universitaria que responda a las nuevas demandas “... más que preocuparse por el manejo instrumental de los conocimientos disponibles; a la universidad se le exige (...) conectar los conocimientos, transmitir nuevas competencias, responder al contexto en un marco de mundialización creciente, y crear aptitudes sólidas para pensar" (Morín, 2005 citado por Sánchez, 2013, p. 14).

Para ello, es imperativo el ejercicio de un liderazgo universitario que pueda transformar el comportamiento de la gente y de la institución universitaria en cuanto a su estructura y funciones. Tanto es así, que líderes han tenido que tomar acciones para transformar el statu quo que ha prevalecido en la educación universitaria para alinearse a estos cambios y elevar las competencias en investigación, desarrollo tecnológico e innovación del personal docente, y que su vez adquieran nuevas herramientas para el aprendizaje permanente de los estudiantes. Ello implicaría asumir un estilo de liderazgo que logre los cambios para tales propósitos, como lo es el liderazgo transformacional, el cual está orientado a valorar el aspecto humano, privilegiando las capacidades de la gente que hace vida en estas instituciones, apalancando el esfuerzo en conjunto mediante procesos que incentiven a las personas para alcanzar los estándares de calidad educativa, producción de conocimientos y desarrollo intelectual que demanda la sociedad en general, sobre todo en escenarios complejos y de incertidumbre.

Salazar (2006); Martins, Carmmaroto, Neris y Canelón (2009); y Bracho y García (2013), enfatizan que el liderazgo transformacional se caracteriza por ser carismático, visionario, transformador, flexible e inclusivo, donde el denominador común es la influencia que se ejerce en las personas para lograr el compromiso 
orientando a una visión. Es una modalidad de liderazgo que busca transformar el comportamiento del conjunto de personas dentro de una institución. Bajo estas premisas, y considerando la importancia del liderazgo transformacional para producir cambios sustanciales, principalmente en aquellos que se están generando en escenarios globales, este artículo tiene como propósito dar a conocer el estado del arte de las tendencias globales en la educación universitaria con una mirada desde el liderazgo transformacional.

\section{Discusión}

Hace unos años se hablaba sobre la resistencia al cambio como algo natural en las personas dentro de las organizaciones, y había que darle tiempo suficiente para que lo pudieran asimilar. Sin embargo, en la actualidad se ha entendido que no tiene sentido resistirse al cambio, más bien hay que aceptarlo porque el cambio llega muy rápido $\mathrm{y}$ muchas veces sin avisar, es inevitable, es permanente. Más bien el cambio ha pasado a ser la norma, en consecuencia si una organización no lidera el cambio, no perdurará. En este tenor, Drucker (2002) sustenta la tesis que ninguna institución, sea un negocio, un hospital o una universidad, puede pretender sobrevivir, mucho menos tener éxito, si no está a la altura de los parámetros fijados por los líderes en su campo en cualquier parte del mundo. Por lo tanto, las instituciones están obligadas a hacer el mayor esfuerzo para ser receptivas al cambio y capaces de cambiar, y un líder del cambio está para promover esos cambios.
El planteamiento anterior resulta oportuno, dada la crisis e incertidumbre que acontece en el mundo en el orden económico, social, político, científico, tecnológico y ecológico luego de la caída del muro de Berlín, entre los que destacan la crisis financiera mundial, la destrucción paulatina del planeta por sus habitantes, la crisis mundial de los alimentos, la crisis mundial de las ideologías y la crisis del poder mundial; colocando en evidencia el resquebrajamiento de las doctrinas neoliberales, la estructura ideológica, espiritual y cultural de la globalización y del capitalismo financiero (Amin, 2000).

Este escenario ha obligado a los países a liderar los cambios y ofrecer soluciones a los grandes problemas que afectan a la humanidad, estableciendo acciones en común para alcanzar objetivos globales, tal como lo señala Sachs (2008, p. 385) quien argumenta que los sectores público y privado, además de los sectores no lucrativos como las fundaciones y las universidades están contribuyendo a la solución de estos problemas que impactan al mundo, destacando las siguientes responsabilidades.

Sector público: Sufragar la investigación científica (...); Promover el desarrollo (...) de tecnologías; Financiar la generalización de innovaciones y tecnologías de éxito. Sector privado: Invertir en $\mathrm{I}+\mathrm{D}(\ldots)$ con financiamiento público; Aplicar soluciones tecnológicas (...) en colaboración con el sector público. Sector no lucrativo: Elevar recomendaciones públicas; Canalizar la iniciativa social y la resolución de 
problemas; (...) Realizar investigación científica, sobre todo en instituciones académicas.

Estas reflexiones colocan en contexto la relevancia de estos sectores de la sociedad para liderar los cambios, entre ellos al sector universitario, el cual tiene como papel esencial para la solución de problemas globales realizar investigaciones científicas -además de las funciones de docencia y extensión-, cuyos temas a abordar respecto a las necesidades en materia de desarrollo sostenible son amplios. A tal efecto, Sachs (2008), enfatiza en los siguientes: a) I+D e innovación para luchar contra enfermedades como el SIDA, la malaria, la tuberculosis y grandes epidemias; b) El calentamiento global y sus implicaciones ambientales como la agricultura, la energía sostenible, las tecnologías de gestión del agua y la conservación de la biodiversidad, entre otras; c) La universalización de los Derechos Humanos; d) La Responsabilidad Social Empresarial, iniciativa que busca involucrar a las organizaciones con la comunidad; e) La producción de bienes y servicios vinculados a redes productivas; y f) El surgimiento de la sociedad del conocimiento como alternativa para generar innovación mediante la $\mathrm{I}+\mathrm{D}$, haciendo a las empresas y países más prósperos y competitivos.

No hay dudas que para liderar los cambios y alcanzar los objetivos globales enmarcados en estos grandes temas a fin de garantizar el desarrollo sostenible son procesos complejos, los cuales sobrepasan las capacidades del sector universitario en muchos países -apoyados de los otros sectores aludidos-, siendo pertinente la creación de redes globales para aprovechar todas las potencialidades en los distintos ámbitos de la sociedad (Stiglitz, 2006).

A tal efecto, uno de los aportes más evidentes que han permitido la instauración de redes globales son las Tecnologías de Información y Comunicación (TIC), las cuales están revolucionando las formas de generar desarrollo con una inversión relativamente baja, ayudando cada vez más a desarticular el aislamiento de países y regiones en su interior, incluyendo lugares remotos con comunidades muy pobres. En este sentido, una de las tantas contribuciones que ofrecen las TIC para preservar un desarrollo sostenible es la educación y formación (Sachs, 2008).

En sí, el aprendizaje a distancia ha sido uno de los mecanismos que ha tenido alto valor utilitario por sus diversas formas de educación y formación que ofrecen las universidades para producir conocimientos. Esta tendencia ha permitido la creación de aulas virtuales, donde los estudiantes pueden interactuar de manera simultánea con otros países a través de plataformas en línea y videoconferencias. Dentro de los ejemplos más notables que simbolizan esta nueva modalidad de desarrollo, es la que se ha puesto en práctica en la India a través de la telemedicina, permitiendo a sus médicos de zonas urbanas atender a pacientes en comunidades rurales más pobres, práctica que está proyectada a la 
atención médica desde la India a pacientes en África y adicionalmente, formar, retroalimentar y orientar por esta vía a los trabajadores sanitarios de zonas rurales africanas (Sachs, 2008).

En consecuencia, las universidades han tenido que producir cambios profundos en su manera de operar para responder a las necesidades que demanda la sociedad, lo cual ha tenido un impacto significativo. En este tenor, autores como Ramírez (2004), Silva (2007) y Rusque (2008) destacan estos cambios: a) Se ha incrementado la flexibilización de la educación, al asumir el reto de incorporar las TIC como herramienta que contribuye a la creación e innovación, con cierta lentitud en sectores conservadores de la sociedad para asimilar estos cambios. Esta oferta educativa ha requerido nuevos modelos pedagógicos para encarar las propuestas de enseñanza presencial, a distancia o mixtas: b) Ha aumentado la descentralización organizacional y la gestión educativa, dándole mayor participación a la comunidad; c) Se usan herramientas de evaluación para medir el desempeño tanto de alumnos como de profesores; d) Se está promoviendo el empleo de profesionales entre 23 y 26 años, acortando la duración del pregrado a tres años, alineado con conocimientos que demanda la sociedad y estimulando la conducción de estudios de maestría y doctorado; e) Se están modificando los currícula, implementando métodos nuevos y adecuados que faciliten la adquisición de técnicas, competencias y capacidades de comunicación, el pensamiento autónomo e independiente, $\mathrm{y}$ el trabajo en equipo, todas ellas con el estímulo a la creatividad combinada con el saber, la ciencia y tecnologías avanzadas; f) Se solicitan más y mejores resultados a las universidades, a pesar que se tiende a otorgar menos presupuesto, donde estas instituciones hacen asociaciones estratégicas con el sector privado en materia de $\mathrm{I}+\mathrm{D}$ y registro de patentes; g) Hay mayor acceso de los estudiantes a los recursos del aprendizaje con una instrucción adaptada a las necesidades específicas de cada uno, relacionadas a sus habilidades, conocimientos e intereses, estimulando el trabajo en equipo para alcanzar objetivos en común y ejecutando tareas de resolución de conflictos. Todo ello con el criterio de prepararlos para los puestos de trabajo a futuro; y h) Presencia de mayor propensión de los profesores a realizar labores que faciliten el aprendizaje, mediante tutorías, coaching y retroalimentación, entre otras, lo que implica menos horas presenciales donde se valora tanto el trabajo individual como en equipo.

\section{Ícono referencial de éxito: el grupo BRIC}

Sin lugar a dudas, los cambios señalados han conducido a que muchos países emergentes, industrializados o en desarrollo no se coloquen de espaldas frente a estas realidades; a tal efecto, han impulsado un liderazgo para producir verdaderas transformaciones en la educación universitaria, tal como lo sostiene Sachs (2008) quien señala que uno de estos países ha sido la India, donde han realizado cambios importantes fijados por los líderes en este sector. En 
esta nación se crearon varias universidades tecnológicas, cuya premisa en su proceso de instauración fue que imitaran a las mejores del mundo y que a su vez, tuvieran convenios con la institución modelo a fin de asegurar transferencia tecnológica. El primer Instituto Indio de Tecnología diseñó su plan de estudios basado en el Massachussetts Institute of Technology (MIT) de los Estados Unidos, con quienes se hizo un convenio para llevar un numeroso grupo de docentes a esa casa de estudios. Posteriormente, se realizaron convenios con universidades tecnológicas de la ex Unión Soviética, Gran Bretaña, entre otras. Esta visión se mantuvo de forma sostenida, y hoy en día la India ha sumado un total de 12 Institutos tecnológicos en distintas ciudades de la India, cada uno de ellos asociados con un país distinto (Oppenheimer, 2010). Es importante destacar que el proceso de ingreso para estudiar en estos institutos es sumamente riguroso, inclusive más exigente que para ingresar al MIT. Oppenheimer también indica que al principio tuvo muchas críticas, pero al tiempo estos cambios generaron resultados que permitieron a la India liderar una economía con nuevos profesionales y técnicos en la ingeniería, y convertirla en uno de los países de mayor crecimiento en el mundo.

Por su parte en China, luego de la Revolución Cultural de Mao, la educación universitaria proporcionaba médicos y músicos de buena calidad; sin embargo, existía ausencia de profesionales con conocimientos en el área gerencial y de negocios. Estas circunstancias los indujeron a implementar tres estrategias para la educación universitaria y alinearse con el resto del mundo.

Estimular las inversiones extranjeras para atraer tecnología internacional y capital humano al país; estimular a que los mejores estudiantes del país hicieran sus licenciaturas y maestrías en el extranjero; e invitar a universidades extranjeras a establecerse en China, primero en forma experimental, y luego de manera definitiva

(Oppenheimer,2010, p. 162).

Así pues, resalta este autor que China realizó alianzas estratégicas con las principales escuelas de negocio del mundo -varios programas patrocinados por la Universidad de Harvard-, con sucursales tanto en China como en Europa y los Estados Unidos. Asimismo, China ha firmado más de mil acuerdos académicos internacionales dentro de su política de internacionalización de la educación, además que 170 universidades extranjeras otorgan títulos válidos en China.

En lo referente a Brasil, Sennes (2009) sintetiza los aportes de este país en materia de educación universitaria de esta forma: (1) Sus estudios de postgrado están catalogados como los de mayor calidad en Latinoamérica, graduando anualmente en promedio 30.000 magísteres y 10.000 doctores; (2) Brasil invierte el $63 \%$ en $\mathrm{I}+\mathrm{D}$ en relación con lo que invierte la región; (3) La cantidad y 
calidad de las publicaciones científicas que produce el sector universitario se equiparan con las de los países de mayor desarrollo. Adicionalmente, Brasil ha establecido alianzas con el sector privado nacional e internacional para incrementar el acceso de estudiantes a la educación universitaria, debido a que el Estado por sí sólo no puede atender la alta demanda.

A la luz de estas consideraciones en torno a estas tres naciones -India, China y Brasil-, se pueden observar los cambios y transformaciones que han realizado en la educación universitaria, estableciendo convenios con líderes mundiales en tecnología, innovación e $\mathrm{I}+\mathrm{D}$, en el área de negocios; y asociaciones estratégicas con el sector privado nacional e internacional. Estos cambios han conducido a estos países que pertenecen al grupo BRICS, a ser las economías emergentes más importantes del mundo, contribuyendo al crecimiento y desarrollo de sus respectivas naciones (Tancredi y Maraschio, 2015)

Como corolario a lo anterior, Stiglitz (2006) enfatiza que la educación en América Latina será la clave para que la región pueda ser competitiva. De igual forma, debe adecuar su estrategia para modernizar sus habilidades, además de invertir y mejorar en ciencia y tecnología, puesto que el mundo es cada vez más competitivo, donde las naciones se están disputando cuotas del mercado. En definitiva, los países están obligados a prepararse, adquirir conocimientos $\mathrm{y}$ habilidades para competir en el mercado mundial.

\section{La educación universitaria desde el liderazgo transformacional}

De acuerdo a los razonamientos que se han venido realizando en torno a las tendencias globales en la educación universitaria, reiteradamente se ha aludido a los descriptores liderazgo, cambios, y transformaciones. Pues sí, estas tendencias han sido producto de un liderazgo que ha llegado más allá de lo que otros no ven, generando profundos cambios, y sobre todo transformaciones en aspectos vitales que afectan a la sociedad; en sí, son tendencias en la educación universitaria que han requerido de un liderazgo transformacional. En efecto, Robbins (2004, p. 343) asevera que el liderazgo transformacional “... logra que los seguidores vean más allá de sus propios intereses y ejerce un profundo y extraordinario efecto sobre ellos".

Por su parte, Lussier y Achua (2008, p. 5) definen al liderazgo transformacional como:

Capacidad para cambiar el statu quo de la organización y una visión convincente de lo que podría ser una nueva organización (...) en consecuencia, buscan transformar o cambiar los valores, creencias o actitudes básicas de los seguidores, de manera que estén dispuestos a desempeñarse más allá de los niveles mínimos especificados por la organización.

En este sentido, se puede inferir que uno de los aspectos cruciales para afianzar los cambios organizacionales -en 
este caso, la educación universitaria-, es a partir de un liderazgo transformacional, $\mathrm{y}$ pasa por transformar al individuo, por transformar la vida de las personas, con énfasis en los rasgos de personalidad -actitudes, creencias, hábitos, pensamientos, valores, filtros, emociones, entre otros-, que por su naturaleza se caracterizan por ser subjetivos dado que no son tangibles, condicionando los patrones de comportamiento del individuo y del conjunto de personas que hacen vida en las universidades. En definitiva, el liderazgo transformacional aplica, si se logra transformar las maneras de pensar, sentir y actuar de la gente (Bass y Riggio, 2006); sólo así podría cambiar sustancialmente la educación universitaria. De acuerdo a ello, las universidades y sus docentes tienen la responsabilidad y el compromiso de contribuir con el desarrollo de la sociedad, adquiriendo competencias de liderazgo para transformar el ámbito académico, mediante procesos de capacitación permanente.

Así pues, un liderazgo transformacional en las universidades implicaría desmarcarse de formas tradicionales de conducirse, acogiendo nuevas estrategias para inspirar a las personas, transmitiendo optimismo y favoreciendo un ambiente propicio para garantizar cambios en la gente (González, González, Ríos, y León, 2013), lo que implicaría la adquisición de habilidades vinculadas a esta modalidad de liderazgo; en sí, un liderazgo transformacional en la educación universitaria que pueda articularse con las tendencias educacionales presentes en la aldea global.

\section{Conclusiones}

La educación universitaria es uno de los sectores de cardinal importancia para consolidar una sólida educación y formación, por ende sería necesario indagar en futuras investigaciones acerca de la brecha que tienen las universidades ecuatorianas con respecto a la educación universitaria en el mundo, a los fines de hacer aquellos cambios que permitan acercarse a las tendencias educacionales que están ocurriendo de manera acelerada y contribuir no sólo a la resolución de problemas globales, sino también al desarrollo la nación. Para tal fin, sería pertinente concebir un liderazgo desde estas instituciones para producir cambios profundos en beneficio de la sociedad, que no es más que el liderazgo transformacional.

Por lo tanto, en una sociedad caracterizada por la complejidad y la permanente incertidumbre, el ejercicio docente necesita de competencias que estén asociadas a esta modalidad de liderazgo, a los fines de promover los cambios que acontecen en la aldea global, transformando a las universidades en centros que faciliten la innovación, la I+D y la transferencia tecnológica en términos de cantidad y calidad, haciendo alianzas estratégicas y convenios con universidades extranjeras de alto nivel académico e investigativo, y consolidando equipos de trabajo articulados con una visión compartida. 
Desde luego que para sustentar lo anterior, es menester que la las universidades en su conjunto asuman de igual modo el ejercicio del liderazgo transformacional, no sólo para modificar sus estructuras, que sería plausible, sino también para influir en los patrones de comportamiento a fin potenciar los cambios entre sus pares, colaboradores y relacionados en cuanto a las maneras de pensar, sentir y actuar, los cuales están condicionadas por sus creencias y sus valores; en definitiva, un liderazgo que permita transformar la concepción que se tiene acerca de la realidad, para alinearla con las tendencias globales en la educación universitaria.

\section{Referencias}

Amin, S. (2000). El capitalismo en la era de la globalización. Barcelona: Editorial Paidos.

Bass, B., y Riggio, R. (2006) Transformational leadership. New Jersey: Lawrence Erlbaum.

Bracho, O., y García, J. (2013). Algunas consideraciones teóricas sobre el liderazgo transformacional. Telos Revista de Estudios Interdisciplinarios en Ciencias Sociales, 15 (2), 165-177.

Drucker, P. (2002). Los Desafios de la Gerencia del Siglo XXI. México: Norma.

González, O., González, O., Ríos, G., y León, J. (2013). Características del liderazgo transformacional presentes en un grupo de docentes universitarios. Telos Revista de Estudios Interdisciplinarios en Ciencias Sociales, 15 (3), 355-371.

Lanz, R., y Ferguson, A. (2011). La transformación universitaria y la relación universidad -estado-mundo. Revista Venezolana de Educación EDUCERE, (50), 177-186. Lussier, R. y Achúa C. (2008). Liderazgo. México: Thomson Learning.

Lussier, R., y Achua, C. (2008). Liderazgo. México: Thomson Learning.

Martins, F., Carmmaroto, A., Neris, L., y Canelón, E. (2009). Liderazgo transformacional y gestión educativa en contextos descentralizados. Revista Actualidades Investigativas en Educación 9 (2), 1-27.

Morín, E. (2001). Introducción al pensamiento complejo. Bogotá: Gedisa.

Oppenheimer, A. (2010). Basta de historias. México: Editorial Debate.

Ramírez, L. (2004). Los nuevos desafíos de la gerencia educativa. Educación $y$ educadores, (7), 113-136.

Robbins, S. (2004). Comportamiento organizacional. México: Pearson Educación.

Rusque, A. (2008). Empresarios y gerentes sujetos en formación en la educación superior. En Silva, J., y Graterol, E. (Ed.), Gerencia y condición humana (9-23). Caracas: Fondo Editorial Tropykos.

Sachs, J. (2008). Economía para un Planeta Abarrotado. Barcelona: Editorial Debate.

Salazar, M. (2006). El Liderazgo Transformacional ¿modelo para organizaciones educativas que aprenden? UNIrevista 1 (3), 1-12.

Sánchez, L. (2013). Complejidad y coopetitividad. Desafíos estratégicos para la transformación universitaria. Centro de Investigación de Ciencias Administrativas $y$ Gerenciales CICAG, 10 (1), (1-16).

Sennes, R. (2009). Innovation in Brasil. Washington: Woodrow Wilson International Center for Scholars. 
Silva, J. (2007). Las empresas asociadas con universidades en parques tecnológicos son más productivas en su investigación. Madrid: Fundación para el conocimiento.

Stiglitz, J. (2006). Cómo hacer que funcione la globalización. Madrid: Editorial Taurus.

Tancredi, E., y Maraschio, M. (2015). La

permanencia de las tendencias globales y la emergencia de nuevas economías en la geografía del comercio internacional. RED Sociales, Revista del Departamento de Ciencias Sociales, 2 (2), 2-32. 